NOTICE: this is the author's version of a work that was accepted for publication in Automatica. Changes resulting from the publishing process, such as peer review, editing, corrections, structural formatting, and other quality control mechanisms may not be reflected in this document. Changes may have been made to this work since it was submitted for publication. A definitive version was subsequently published in Automatica, Vol. 49, No. 2 (2013).

DOI: 10.1016/j.automatica.2012.11.006 


\title{
The Generalised Discrete Algebraic Riccati Equation in Linear-Quadratic optimal control ${ }^{\star}$
}

\author{
Augusto Ferrante ${ }^{a}$, Lorenzo Ntogramatzidis ${ }^{b}$ \\ ${ }^{\text {a} D i p a r t i m e n t o ~ d i ~ I n g e g n e r i a ~ d e l l ' I n f o r m a z i o n e, ~ U n i v e r s i t a ̀ ~ d i ~ P a d o v a, ~ v i a ~ G r a d e n i g o, ~ 6 / B ~-~} 35131$ Padova, Italy. \\ Research carried out while visiting Curtin University, Perth (WA), Australia. \\ ${ }^{\mathrm{b}}$ Department of Mathematics and Statistics, Curtin University, Perth (WA), Australia.
}

\begin{abstract}
This paper investigates the properties of the solutions of the generalised discrete algebraic Riccati equation arising from the classic infinitehorizon linear quadratic (LQ) control problem. In particular, a geometric analysis is used to study the relationship existing between the solutions of the generalised Riccati equation and the output-nulling subspaces of the underlying system and the corresponding reachability subspaces. This analysis reveals the presence of a subspace that plays an important role in the solution of the related optimal control problem, which is reflected in the generalised eigenstructure of the corresponding extended symplectic pencil. In establishing the main results of this paper, several ancillary problems on the discrete Lyapunov equation and spectral factorisation are also addressed and solved.
\end{abstract}

Key words: Generalised discrete algebraic Riccati equation, LQ optimal control, output-nulling subspaces, reachability subspaces.

\section{Introduction}

Due to their ubiquitousness in optimal control and filtering problems, as well as in linear factorisation and stochastic realisation problems, Riccati equations are universally regarded as a cornerstone of modern control theory. Several monographs have been entirely devoted to providing a general and systematic framework for the study of Riccati equations, see e.g. $[8,7,1]$.

The classic solution of the discrete-time infinite-horizon LQ problem is traditionally expressed in terms of the solution $X$ of the Riccati equation

$X=A^{\mathrm{T}} X A-\left(A^{\mathrm{T}} X B+S\right)\left(R+B^{\mathrm{T}} X B\right)^{-1}\left(B^{\mathrm{T}} X A+S^{\mathrm{T}}\right)+Q$,

where $A \in \mathbb{R}^{n \times n}, B \in \mathbb{R}^{n \times m}, Q \in \mathbb{R}^{n \times n}, S \in \mathbb{R}^{n \times m}$ and $R \in$

\footnotetext{
* Partially supported by the Italian Ministry for Education and Research (MIUR) under PRIN grant n. 20085FFJ2Z and by the Australian Research Council under the grant FT120100604. Corresponding author L. Ntogramatzidis. Tel. +61-8-92663143.
}

Email addresses: augusto@dei.unipd.it (Augusto Ferrante), L. Ntogramatzidis@curtin.edu.au (Lorenzo Ntogramatzidis).
$\mathbb{R}^{m \times m}$ are such that the Popov matrix $\Pi$ satisfies

$$
\Pi \stackrel{\text { def }}{=}\left[\begin{array}{cc}
Q & S \\
S^{\mathrm{T}} & R
\end{array}\right]=\Pi^{\mathrm{T}} \geq 0 .
$$

The set of matrices $\Sigma=(A, B ; Q, R, S)$ is often referred to as Popov triple, see e.g. [7]. Equation (1) is the so-called Discrete Riccati Algebraic Equation $\operatorname{DARE}(\Sigma)$.

Nevertheless, an LQ problem may have solutions even if $\operatorname{DARE}(\Sigma)$ has no solutions, and the optimal control can be written in this case as a state feedback given in terms of a matrix $X$ such that $R+B^{\mathrm{T}} X B$ is singular and satisfies the more general Riccati equation

$$
\begin{gathered}
X=A^{\mathrm{T}} X A-\left(A^{\mathrm{T}} X B+S\right)\left(R+B^{\mathrm{T}} X B\right)^{\dagger}\left(B^{\mathrm{T}} X A+S^{\mathrm{T}}\right)+Q \\
\operatorname{ker}\left(R+B^{\mathrm{T}} X B\right) \subseteq \operatorname{ker}\left(A^{\mathrm{T}} X B+S\right)
\end{gathered}
$$

where the matrix inverse in $\operatorname{DARE}(\Sigma)$ has been replaced by the Moore-Penrose pseudo-inverse, see [10]. Eq. (3) is known as the generalised discrete-time algebraic Riccati equation $\operatorname{GDARE}(\Sigma)$. The $\operatorname{GDARE}(\Sigma)$ with the additional constraint (4) is sometimes referred to as constrained generalised discrete-time algebraic Riccati equation, herein denoted by $\operatorname{CGDARE}(\Sigma)$. It is obvious that (3) is a generalisation of the classic $\operatorname{DARE}(\Sigma)$, in the sense that any solution of $\operatorname{DARE}(\Sigma)$ is also a solution of $\operatorname{GDARE}(\Sigma)$ - and therefore 
also of CGDARE $(\Sigma)$ because $\operatorname{ker}\left(R+B^{\mathrm{T}} X B\right)=0_{m}$ - but the vice-versa is not true in general. Despite its generality, this type of Riccati equation has only been marginally studied in the monographs $[11,7,1]$ and in the paper [3]. The only contributions entirely devoted to the study of the solutions of this equation are [6] and [12]. The former investigates conditions under which the $\operatorname{GDARE}(\Sigma)$ admits a stabilising solution in terms of the deflating subspaces of the extended symplectic pencil. The latter studies the connection between the solutions of this equation and the rank-minimising solutions of the so-called Riccati linear matrix inequality. In pursuing this task, the authors of [12] derived a series of results that shed some light into the fundamental role played by the term $R_{X} \stackrel{\text { def }}{=} R+B^{\mathrm{T}} X B$. An example is the important observation according to which the inertia of this matrix $R_{X}$ is independent of the solution $X$ of $\operatorname{CGDARE}(\Sigma)$, [12, Theorem 2.4]. Hence, $\mathbf{i})$ if $X$ is a solution of $\operatorname{DARE}(\Sigma)$, then all solutions of $\operatorname{CGDARE}(\Sigma)$ will also satisfy $\operatorname{DARE}(\Sigma)$ and, ii) if $X$ is a solution of $\operatorname{CGDARE}(\Sigma)$ such that $R_{X}$ is singular, then DARE $(\Sigma)$ does not admit solutions. The results presented in [12] are established in the very general setting in which the Popov matrix $\Pi$ is not necessarily positive semidefinite as in (2).

It is often taken for granted that $\operatorname{GDARE}(\Sigma)$ generalises the standard $\operatorname{DARE}(\Sigma)$ in the solution of the infinite LQ optimal control problem in the same way in which [10] established that the generalised Riccati difference equation generalises the standard Riccati difference equation in the solution of the finite-horizon LQ problem. However, to the best of the authors' knowledge, this fact has never been presented in a direct, self-contained and rigorous way. Thus, the first aim of this paper is to show the connection of the $\operatorname{CGDARE}(\Sigma)$ and the solution of the standard infinite-horizon LQ optimal control problem. The second aim of this paper is to provide a geometric picture describing the structure of the solutions of the CGDARE $(\Sigma)$ in terms of the output nulling subspaces and the corresponding reachability subspaces of $\Sigma$. Indeed, when $\Pi \geq 0$, the null-space of $R_{X}$ is independent of the solution $X$ of $\operatorname{CGDARE}(\Sigma)$, and is linked to the presence of a subspace which plays an important role in the characterisation of the solutions of $\operatorname{CGDARE}(\Sigma)$ and in the solution of the related optimal control problem. This subspace does not depend on the particular solution $X$, nor does the closedloop matrix restricted to this subspace. This new geometric analysis reveals that the spectrum of the closed-loop system is divided into a part that depends on the solution $X$ of $\operatorname{CGDARE}(\Sigma)$, and one - coinciding with the eigenvalues of the closed-loop restricted to this subspace - which is independent of it. At first sight, this fact seems to constitute a limitation in the design of the optimal feedback, because regardless of the solution of the generalised Riccati equation chosen for the implementation of the optimal feedback, the closed-loop matrix will always present a certain fixed eigenstructure as part of its spectrum. However, when $R_{X}$ is singular, the set of optimal controls presents a further degree of freedom - which is also identified in [11, Remark 4.2.3] - that allows to place all the closed-loop poles at the desired locations without changing the cost.

\section{Linear Quadratic optimal control and CGDARE}

In this section we analyse the connections between LQ optimal control and CGDARE. Most of the results presented here are considered "common wisdom". However, we have not been able to find a place where they have been explicitly derived, so we believe that this section may be useful. Consider the discrete linear time-invariant system governed by

$$
x_{t+1}=A x_{t}+B u_{t},
$$

where $A \in \mathbb{R}^{n \times n}$ and $B \in \mathbb{R}^{n \times m}$, and let the initial state $x_{0} \in$ $\mathbb{R}^{n}$ be given. The problem is to find a sequence of inputs $u_{t}$, with $t=0,1, \ldots, \infty$, minimising the cost function

$$
J\left(x_{0}, u\right) \stackrel{\text { def }}{=} \sum_{t=0}^{\infty}\left[\begin{array}{ll}
x_{t}^{\mathrm{T}} & u_{t}^{\mathrm{T}}
\end{array}\right]\left[\begin{array}{cc}
Q & S \\
S^{\mathrm{T}} & R
\end{array}\right]\left[\begin{array}{l}
x_{t} \\
u_{t}
\end{array}\right] .
$$

Before we introduce the solution of the optimal control problem, we recall some well-known classic linear algebra results which will be useful in the sequel, see e.g. $[3,5]$.

Lemma 2.1 Consider the symmetric positive semidefinite matrix $P=\left[\begin{array}{ll}P_{11} & P_{12} \\ P_{12}^{T} & P_{22}\end{array}\right]$. Then,

(i) $\operatorname{ker} P_{12} \supseteq \operatorname{ker} P_{22}$,

(iii) $P_{12}\left(I-P_{22}^{\dagger} P_{22}\right)=0$,

(ii) $P_{12} P_{22}^{\dagger} P_{22}=P_{12}$,

(iv) $P_{11}-P_{12} P_{22}^{\dagger} P_{12}^{T} \geq 0$.

Given $X=X^{\mathrm{T}} \in \mathbb{R}^{n \times n}$ we define

$Q_{X} \stackrel{\text { def }}{=} Q+A^{\mathrm{T}} X A-X, S_{X} \stackrel{\text { def }}{=} A^{\mathrm{T}} X B+S, R_{X} \stackrel{\text { def }}{=} R+B^{\mathrm{T}} X B$, (7)

$G_{X} \stackrel{\text { def }}{=} I_{m}-\left(R+B^{\mathrm{T}} X B\right)^{\dagger}\left(R+B^{\mathrm{T}} X B\right)=I_{m}-R_{X}^{\dagger} R_{X}$,

$K_{X} \stackrel{\text { def }}{=}\left(R+B^{\mathrm{T}} X B\right)^{\dagger}\left(B^{\mathrm{T}} X A+S^{\mathrm{T}}\right)=R_{X}^{\dagger} S_{X}^{\mathrm{T}}$,

$A_{X} \stackrel{\text { def }}{=} A-B\left(R+B^{\mathrm{T}} X B\right)^{\dagger}\left(B^{\mathrm{T}} X A+S^{\mathrm{T}}\right)=A-B K_{X}$,

$\Pi_{X} \stackrel{\text { def }}{=}\left[\begin{array}{ll}Q_{X} & S_{X} \\ S_{X}^{\mathrm{T}} & R_{X}\end{array}\right]$.

The term $R_{X}^{\dagger} R_{X}$ is the orthogonal projector that projects onto $\operatorname{im} R_{X}^{\dagger}=\operatorname{im} R_{X}$ so that $G_{X}$ is the orthogonal projector that projects onto $\operatorname{ker} R_{X}$. Hence, $\operatorname{ker} R_{X}=\operatorname{im} G_{X}$. When $X$ is a solution of $\operatorname{CGDARE}(\Sigma)$, then $K_{X}$ is the corresponding gain matrix, $A_{X}$ the associated closed-loop matrix, and $\Pi_{X}$ is the so-called dissipation matrix. All symmetric and positive semidefinite solutions of $\operatorname{GDARE}(\Sigma)$ satisfy (4), and are therefore solutions of $\operatorname{CGDARE}(\Sigma)$. In fact, if $X \geq 0$, we find $\left[\begin{array}{cc}Q_{X}+X & S_{X} \\ S_{X}^{\mathrm{T}} & R_{X}\end{array}\right]=\left[\begin{array}{c}A^{\mathrm{T}} \\ B^{\mathrm{T}}\end{array}\right] X\left[\begin{array}{ll}A & B\end{array}\right]+\Pi \geq 0$. Therefore, applying Lemma 2.1 we find (4), that can be rewritten as $\operatorname{ker} R_{X} \subseteq \operatorname{ker} S_{X}$ and also as $S_{X} G_{X}=0$. 
Theorem 2.1 Suppose that for every $x_{0}$ there exists an input $u_{t} \in \mathbb{R}^{m}$, with $t \in \mathbb{N}$, such that $J\left(x_{0}, u\right)$ is finite. Then we have:

(1) CGDARE( $\Sigma)$ admits symmetric solutions: a solution $\bar{X}=\bar{X}^{T} \geq 0$ may be obtained as the limit of the sequence of matrices generated by iterating the generalised Riccati difference equation (see (14)) with zero initial condition.

(2) The value of the optimal cost is $x_{0}^{\mathrm{T}} \bar{X} x_{0}$.

(3) $\bar{X}$ is the minimum positive semidefinite solution of CGDARE $(\Sigma)$.

(4) The set of all optimal controls minimising (6) is

$$
u_{t}=-K_{\bar{X}} x_{t}+G_{\bar{X}} v_{t}, \quad v_{t} \text { arbitrary } .
$$

Proof: (1). Consider the finite horizon LQ problem consisting in the minimisation of the performance index

$$
J_{T} \stackrel{\text { def }}{=} \sum_{t=0}^{T-1}\left[\begin{array}{ll}
x_{t}^{\mathrm{T}} & u_{t}^{\mathrm{T}}
\end{array}\right] \Pi\left[\begin{array}{l}
x_{t} \\
u_{t}
\end{array}\right]
$$

subject to (5) with assigned initial state $x_{0} \in \mathbb{R}^{n}$. The optimal control is obtained by iterating, backward in time starting from the terminal condition $P_{T}(T)=0$, the generalised Riccati difference equation $P_{T}(t)=\mathbf{R}\left[P_{T}(t+1)\right]$, [10], where $\mathbf{R}[\cdot]$ is the Riccati operator defined as

$\mathbf{R}[P] \stackrel{\text { def }}{=} A^{\mathrm{T}} P A-\left(A^{\mathrm{T}} P B+S\right)\left(R+B^{\mathrm{T}} P B\right)^{\dagger}\left(B^{\mathrm{T}} P A+S^{\mathrm{T}}\right)+Q$

and the optimal value of the cost is $J_{T}^{*}\left(x_{0}\right)=x_{0}^{\mathrm{T}} P_{T}(0) x_{0}$. Consider the "reverse time" sequence of matrices defined as $X_{t} \stackrel{\text { def }}{=} P_{t}(0)$. Since $P_{\tau}(t)=P_{\tau-t}(0)$ for all $t \leq \tau$, the sequence $\left\{X_{t}\right\}_{t \in \mathbb{N}}$ is obtained by iterating the generalised Riccati difference equation forward with initial condition $X_{0}=0$. The sequence $\left\{J_{t}^{*}\left(x_{0}\right) \stackrel{\text { def }}{=} x_{0}^{\mathrm{T}} X_{t} x_{0}\right\}_{t \in \mathbb{N}}$ is obviously monotonically non-decreasing (it is the sequence of optimal costs over intervals of increasing lengths $t$ ). Hence, $\left\{X_{t}\right\}_{t \in \mathbb{N}}$, and $\left\{R+B^{\mathrm{T}} X_{t} B\right\}_{t \in \mathbb{N}}$ are monotonically non-decreasing sequences of positive semidefinite matrices. We now show that these sequences are bounded. Assume, by contradiction, that $\lim _{t \rightarrow+\infty}\left\|X_{t}\right\|=+\infty$. The sequence $\left\{X_{t}^{1}=\frac{X_{t}}{\left\|X_{t}\right\|}\right\}_{t \in \mathbb{N}}$ is bounded. Thus, there exists a converging sub-sequence $\left\{X_{t_{i}}^{1}\right\}$. Let $\bar{X}^{1}$ be its limit. Clearly $\left\|\bar{X}^{1}\right\|=1$ : let $x_{0}^{1} \in \mathbb{R}^{n}$ be such that $\left\|x_{0}^{1}\right\|=1$ and $\left(x_{0}^{1}\right)^{\mathrm{T}} \bar{X}^{1} x_{0}^{1}=1$. Since we assumed that for any $x_{0}$ there exists a trajectory that renders $J$ in (6) finite, there exist a constant $m_{0}$ and an input trajectory $u^{1}$ such that $J_{t_{i}}^{*}\left(x_{0}^{1}\right) \leq J\left(x_{0}^{1}, u^{1}\right) \leq m_{0}$, where the first inequality follows from the optimality of the cost $J_{t_{i}}^{*}\left(x_{0}^{1}\right)$ and the fact that, for a given $u^{1}$, the index (6) is a sum of infinite nonnegative terms which is greater than or equal to the sum of the first $t_{i}$ terms of the sum. On the other hand we have $J_{t_{i}}^{*}\left(x_{0}^{1}\right)=\left\|X_{t_{i}}\right\|\left(x_{0}^{1}\right)^{\mathrm{T}} X_{t_{i}}^{1} x_{0}^{1} \rightarrow+\infty$, which is a contradiction.

Since $\left\{X_{t}\right\}_{t \in \mathbb{N}}$ is non-decreasing and bounded, it admits a limit $\bar{X}$ for $t \rightarrow \infty$. Then, $\lim _{t \rightarrow \infty} X_{t}=\lim _{t \rightarrow \infty} X_{t+1}=$ $\lim _{t \rightarrow \infty} \mathbf{R}\left[X_{t}\right]=\bar{X}$. Thus, if $\lim _{t \rightarrow \infty} \mathbf{R}\left[X_{t}\right]=\mathbf{R}[\bar{X}]$, then $\mathbf{R}[\bar{X}]=\bar{X}$, i.e. $\bar{X}$ is a positive semidefinite solution of $\operatorname{CGDARE}(\Sigma)$. To prove that this is indeed the case, it is sufficient to show that $\lim _{t \rightarrow \infty} R_{X_{t}}^{\dagger}=R_{\bar{X}}^{\dagger}$. In fact, the pseudoinverse is the only possible source of discontinuity in the Riccati iteration. To prove the latter equality, consider the sequence $\left\{R+B^{\mathrm{T}} X_{t} B\right\}_{t \in \mathbb{N}}$. Since it is a monotonically nondecreasing sequence of positive semidefinite matrices, the chain of inclusions

$\operatorname{ker}\left(R+B^{\mathrm{T}} X_{0} B\right) \supseteq \operatorname{ker}\left(R+B^{\mathrm{T}} X_{1} B\right) \supseteq \operatorname{ker}\left(R+B^{\mathrm{T}} X_{2} B\right) \supseteq \ldots$

holds. Clearly, there exist a $\bar{t}$ such that for any $t \geq \bar{t}$ this chain becomes stationary, i.e., for any $t \geq \bar{t}$ there holds $\operatorname{ker}\left(R+B^{\mathrm{T}} X_{t} B\right)=\operatorname{ker}\left(R+B^{\mathrm{T}} X_{t+1} B\right)$. This implies that a change of coordinates independent of $t$ exists such that in the new basis $R_{X_{t}}=R+B^{\mathrm{T}} X_{t} B=\operatorname{diag}\left\{R_{t}^{0}, O\right\}$, where $\left\{R_{t}^{0}\right\}_{t \geq \bar{t}}$, is a non-decreasing sequence of positive definite matrices. Clearly, $\lim _{t \rightarrow \infty} R_{X_{t}}=R_{\bar{X}}$, so that, in this basis, $R_{\bar{X}}$ has the form $R_{\bar{X}}=R+B^{\mathrm{T}} \bar{X} B=\operatorname{diag}\left\{R^{0}, O\right\}$, where $R^{0} \stackrel{\text { def }}{=} \lim _{t \rightarrow \infty} R_{t}^{0}$. Moreover, since the sequence $\left\{R_{t}^{0}\right\}$ is nondecreasing, $R^{0}$ is also nonsingular, so that $\left(R_{t}^{0}\right)^{-1} \rightarrow\left(R^{0}\right)^{-1}$. Thus, in the chosen basis we have indeed

$R_{X_{t}}^{\dagger}=\left(R+B^{\mathrm{T}} X_{t} B\right)^{\dagger}=\left[\begin{array}{cc}\left(R_{t}^{0}\right)^{-1} & O \\ O & O\end{array}\right] \longrightarrow\left[\begin{array}{cc}\left(R^{0}\right)^{-1} & O \\ O & O\end{array}\right]=R_{\bar{X}}^{\dagger}$.

(2). Let

$$
J^{\circ}\left(x_{0}\right) \stackrel{\text { def }}{=} \inf _{u} J\left(x_{0}, u\right) .
$$

Clearly, $J^{\circ}\left(x_{0}\right) \geq J_{t}^{*}\left(x_{0}\right)=x_{0}^{\mathrm{T}} X_{t} x_{0}$. Then, by taking the limit, we get $J^{\circ}\left(x_{0}\right) \geq x_{0}^{\mathrm{T}} \bar{X} x_{0}$. We now show that the time-invariant feedback control $u_{t}^{*} \stackrel{\text { def }}{=}-K_{\bar{X}} x_{t}$ yields the cost $x_{0}^{\mathrm{T}} \bar{X} x_{0}$, which is therefore the optimal value of the cost. Consider the cost index $J_{T, \bar{X}} \stackrel{\text { def }}{=} J_{T}+x_{T}^{\mathrm{T}} \bar{X} x_{T}$, where $J_{T}$ is defined in (13). It follows from [10, Section II], that an optimal control for this index is given by the time-invariant feedback $u_{t}^{*}=-K_{\bar{X}} x_{t}$ and the optimal cost does not depend on the length $T$ of the time interval and is given by $J_{T, \bar{X}}^{*}=x_{0}^{\mathrm{T}} \bar{X} x_{0}$. For this conclusion we only need the fact that $\bar{X}$ is a positive semidefinite solution of $\operatorname{CGDARE}(\Sigma)$. Now we have

$$
\begin{aligned}
x_{0}^{\mathrm{T}} \bar{X} x_{0} & \leq J^{\circ}\left(x_{0}\right) \leq J\left(x_{0}, u^{*}\right)=\sum_{t=0}^{\infty}\left[x_{t}^{\mathrm{T}}\left(u_{t}^{*}\right)^{\mathrm{T}}\right] \Pi\left[\begin{array}{c}
x_{t} \\
u_{t}^{*}
\end{array}\right] \\
& =\lim _{T \rightarrow \infty} \sum_{t=0}^{T}\left[x_{t}^{\mathrm{T}}\left(u_{t}^{*}\right)^{\mathrm{T}}\right] \Pi\left[\begin{array}{c}
x_{t} \\
u_{t}^{*}
\end{array}\right] \\
& =\lim _{T \rightarrow \infty} J_{T, \bar{X}}^{*}-x_{T}^{\mathrm{T}} \bar{X} x_{T} \leq \lim _{T \rightarrow \infty} x_{0}^{\mathrm{T}} \bar{X} x_{0}=x_{0}^{\mathrm{T}} \bar{X} x_{0} .
\end{aligned}
$$

Comparing the first and last term of the latter expression we see that all the inequalities are indeed equalities, so that the infimum in (15) is a minimum and its value is indeed $x_{0}^{\mathrm{T}} \bar{X} x_{0}$.

(3). Suppose by contradiction that there exist another positive semidefinite solution $\tilde{X}$ of $\operatorname{CGDARE}(\Sigma)$ and a vector $x_{0} \in \mathbb{R}^{n}$ such that $x_{0}^{\mathrm{T}} \tilde{X} x_{0}<x_{0}^{\mathrm{T}} \bar{X} x_{0}$. Take the time-invariant feedback $\tilde{u}_{t}=-K_{\tilde{X}} x_{t}$. The same argument that led to (16) now gives $J\left(x_{0}, \tilde{u}\right) \leq x_{0}^{\mathrm{T}} \tilde{X} x_{0}<x_{0}^{\mathrm{T}} \bar{X} x_{0}$, which is a contradiction because we have shown that $x_{0}^{\mathrm{T}} \bar{X} x_{0}$ is the optimal value of the cost function $J$. 
(4). Let $\mathscr{U}_{0}$ be the set of optimal control inputs at time $t=0$. Let $u_{0} \in \mathbb{R}^{m}$ and $x_{1}=A x_{0}+B u_{0}$ be the corresponding state at $t=1$. Clearly the optimal cost can be written as

$$
J^{*}=x_{0}^{\mathrm{T}} \bar{X} x_{0}=\left[\begin{array}{ll}
x_{0}^{\mathrm{T}} & u_{0}^{\mathrm{T}}
\end{array}\right]\left[\begin{array}{ll}
\bar{X} & O \\
O & O
\end{array}\right]\left[\begin{array}{l}
x_{0} \\
u_{0}
\end{array}\right] .
$$

Moreover, $u_{0} \in \mathscr{U}_{0}$ if and only if the optimal cost can be written in the following alternative form:

$$
\begin{aligned}
J^{*} & =x_{1}^{\mathrm{T}} \bar{X} x_{1}+\left[\begin{array}{ll}
x_{0}^{\mathrm{T}} & u_{0}^{\mathrm{T}}
\end{array}\right] \Pi\left[\begin{array}{l}
x_{0} \\
u_{0}
\end{array}\right] \\
& =\left[\begin{array}{ll}
x_{0}^{\mathrm{T}} & u_{0}^{\mathrm{T}}
\end{array}\right]\left(\left[\begin{array}{l}
A^{\mathrm{T}} \\
B^{\mathrm{T}}
\end{array}\right] \bar{X}\left[\begin{array}{ll}
A & B
\end{array}\right]+\Pi\right)\left[\begin{array}{l}
x_{0} \\
u_{0}
\end{array}\right] .
\end{aligned}
$$

By subtracting the first expression from the second, we get that $u_{0} \in \mathscr{U}_{0}$ if and only if $\left[\begin{array}{ll}x_{0}^{\mathrm{T}} & u_{0}^{\mathrm{T}}\end{array}\right]\left[\begin{array}{ll}Q_{\bar{X}} & S_{\bar{X}} \\ S_{\bar{X}}^{\mathrm{T}} & R_{\bar{X}}\end{array}\right]\left[\begin{array}{l}x_{0} \\ u_{0}\end{array}\right]=0$. Since $\bar{X}$, and hence $\Pi_{\bar{X}}=\left[\begin{array}{cc}Q_{\bar{X}} & S_{\bar{X}} \\ S_{\bar{X}}^{T} & R_{\bar{X}}\end{array}\right]$, are positive semidefinite, this is equivalent to $\left[\begin{array}{ll}Q_{\bar{X}} & S_{\bar{X}} \\ S_{\bar{X}}^{T} & R_{\bar{X}}\end{array}\right]\left[\begin{array}{l}x_{0} \\ u_{0}\end{array}\right]=0$. Finally, this is equivalent to $u_{0}=-R_{\bar{X}}^{\dagger} S_{\bar{X}}^{\mathrm{T}} x_{0}+G_{\bar{X}} v_{0}$, where $v_{0} \in \mathbb{R}^{m}$ is arbitrary, because the columns of $G_{\bar{X}}$ form a basis for $\operatorname{ker} R_{\bar{X}}$. By iterating this argument for all $t=1,2, \ldots$, we get (12).

\section{Preliminary technical results}

In this section, we present several technical results of independent interest that will be used in the sequel.

\subsection{The Hermitian Stein equation}

In this section, we give some important results on the solutions $X$ of the so-called Hermitian Stein equation (known also as the discrete-time Lyapunov equation):

$$
X=A^{\mathrm{T}} X A+Q
$$

where $A, Q \in \mathbb{R}^{n \times n}$ and $Q=Q^{\mathrm{T}} \geq 0$.

Lemma 3.1 Let $X$ be a solution of the Hermitian Stein equation (17). Then, $\operatorname{ker} X$ is A-invariant and is contained in the null-space of $Q$.

Proof: Let $\lambda \in \mathbb{C}$ be on the unit circle and such that $(A+$ $\left.\lambda I_{n}\right)$ is invertible. We can re-write (17) as $X=A^{\mathrm{T}} X(A+$ $\left.\lambda I_{n}\right)-\lambda A^{\mathrm{T}} X+Q$, so that $\left(\lambda A^{\mathrm{T}}+I_{n}\right) X=\lambda A^{\mathrm{T}} X\left(\lambda^{*} A+\right.$ $\left.I_{n}\right)+Q$, since $\lambda$ is on the unit circle (which implies that $\left.\lambda^{*}=\lambda^{-1}\right)$. This is equivalent to

$$
\begin{aligned}
& X\left(\lambda^{*} A+I_{n}\right)^{-1} \\
& =\lambda\left(\lambda A^{\mathrm{T}}+I_{n}\right)^{-1} A^{\mathrm{T}} X+\left(\lambda A^{\mathrm{T}}+I_{n}\right)^{-1} Q\left(\lambda^{*} A+I_{n}\right)^{-1} .
\end{aligned}
$$

Let $\xi \in \operatorname{ker} X$. On pre-multiplying (18) by $\xi^{*}$ and postmultiplying it by $\xi$, we obtain $\xi^{*}\left(\lambda A^{\mathrm{T}}+I_{n}\right)^{-1} Q\left(\lambda^{*} A+\right.$ $\left.I_{n}\right)^{-1} \xi=0$, and since $\left(\lambda A^{\mathrm{T}}+I_{n}\right)^{-1} Q\left(\lambda^{*} A+I_{n}\right)^{-1}$ is Hermitian and positive semidefinite, we get

$$
Q\left(\lambda^{*} A+I_{n}\right)^{-1} \xi=0 .
$$

By post-multiplying (18) by $\xi$, we get $X\left(\lambda^{*} A+I_{n}\right)^{-1} \xi=0$, which means that $\operatorname{ker} X$ is $\left(\lambda^{*} A+I_{n}\right)^{-1}$-invariant. Hence, it is also $\left(\lambda^{*} A+I_{n}\right)$-invariant and therefore $A$-invariant. In view of (19), $\operatorname{ker} X=\left(\lambda^{*} A+I_{n}\right)^{-1} \operatorname{ker} X$ is also contained in $\operatorname{ker} Q$.

We recall that equation (17) has a unique solution if and only if $A$ is unmixed, i.e. for all pairs $\lambda_{1}, \lambda_{2} \in \sigma(A)$ we have $\lambda_{1} \lambda_{2} \neq 1$. In this case, we have (see [14]):

Lemma 3.2 (Lemma 5.1 in [14]) . Let $A$ be unmixed and $X$ be the unique solution of (17) where $Q=Q^{T} \geq 0$. Then, $\operatorname{ker} X$ is the unobservable subspace of the pair $(A, Q)$.

Lemma 3.3 Let $A \in \mathbb{R}^{n \times n}, F \in \mathbb{R}^{n \times n}, B \in \mathbb{R}^{n \times m}$ and assume $X \in \mathbb{R}^{n \times n}$ is such that

$$
\left[\begin{array}{l}
A^{\mathrm{T}} \\
B^{\mathrm{T}}
\end{array}\right] X F=\left[\begin{array}{l}
X \\
O
\end{array}\right]
$$

Then, $B^{\mathrm{T}}\left(A^{\mathrm{T}}\right)^{k} X=0$ for all $k \geq 0$, i.e., $\operatorname{im} X$ is contained in the unobservable subspace of the pair $\left(A^{\mathrm{T}}, B^{\mathrm{T}}\right)$.

Proof: We first prove that $B^{\mathrm{T}} X=0$. Let us choose a basis in which $F=\operatorname{diag}\left\{N, F_{I}\right\}$, where $N$ is nilpotent and $F_{I}$ is invertible. Let us decompose $X$ accordingly, i.e., $X=$ $\left[\begin{array}{ll}X_{1} & X_{2}\end{array}\right]$. Then, $A^{\mathrm{T}} X_{1} N=X_{1}$ implies $X_{1}=0$. In fact, multiplying such equation by $A^{\mathrm{T}}$ and $N$ to the left and to the right, respectively, we obtain $X_{1}=\left(A^{\mathrm{T}}\right)^{k} X_{1} N^{k}$ for all $k \geq 0$. Choosing $k$ to be greater than the nilpotency index of $N$, we get $X_{1}=\left(A^{\mathrm{T}}\right)^{k} X_{1} N^{k}=0$. From (20) we also obtain $B^{\mathrm{T}} X_{2} F_{I}=0$, which gives $B^{\mathrm{T}} X_{2}=0$ since $F_{I}$ is invertible. Thus, $B^{\mathrm{T}} X=0$. The same argument can be iterated to prove that $B^{\mathrm{T}}\left(A^{\mathrm{T}}\right)^{k} X=0$ for all $k \geq 0$. Indeed, by pre-multiplying the first of (20) by $A^{\mathrm{T}}$ we get $A^{\mathrm{T}}\left(A^{\mathrm{T}} X\right) F=A^{\mathrm{T}} X$. By premultiplying the same equation by $B^{\mathrm{T}}$, we get $B^{\mathrm{T}}\left(A^{\mathrm{T}} X\right) F=0$ since $B^{\mathrm{T}} X=0$. Hence, we can write these two equations as $\left[\begin{array}{c}A^{\mathrm{T}} \\ B^{\mathrm{T}}\end{array}\right]\left(A^{\mathrm{T}} X\right) F=\left[\begin{array}{c}A^{\mathrm{T}} X \\ O\end{array}\right]$ and re-apply the same argument used above to show that $B^{\mathrm{T}} A^{\mathrm{T}} X=0$, and so on.

\subsection{Spectral Factorisation}

Since $\Pi=\Pi^{\mathrm{T}} \geq 0$, we can consider the factorisation

$$
\Pi=\left[\begin{array}{cc}
Q & S \\
S^{\mathrm{T}} & R
\end{array}\right]=\left[\begin{array}{l}
C^{\mathrm{T}} \\
D^{\mathrm{T}}
\end{array}\right]\left[\begin{array}{ll}
C & D
\end{array}\right],
$$

where $Q=C^{\mathrm{T}} C, S=C^{\mathrm{T}} D$ and $R=D^{\mathrm{T}} D$. Let us define the rational matrix $W(z) \stackrel{\text { def }}{=} C\left(z I_{n}-A\right)^{-1} B+D$. The spectrum 
(or Popov function) $\Phi(z) \stackrel{\text { def }}{=} W^{\sim}(z) W(z)-$ where $W^{\sim}(z) \stackrel{\text { def }}{=}$ $W^{\mathrm{T}}\left(z^{-1}\right)$ - associated with $\Sigma$ can be written as

$\Phi(z)=\left[B^{\mathrm{T}}\left(z^{-1} I_{n}-A^{\mathrm{T}}\right)^{-1} I_{n}\right]\left[\begin{array}{cc}Q & S \\ S^{\mathrm{T}} & R\end{array}\right]\left[\begin{array}{c}\left(z I_{n}-A\right)^{-1} B \\ I_{n}\end{array}\right]$.

The matrix inequality for an unknown matrix $X=X^{\mathrm{T}}$ of the form $\Pi_{X} \geq 0$ is called the discrete linear matrix inequality, and is denoted by $\operatorname{DLMI}(\Sigma)$. Let us define

$$
L(X) \stackrel{\text { def }}{=} \Pi_{X}-\Pi=\left[\begin{array}{cc}
A^{\mathrm{T}} X A-X & A^{\mathrm{T}} X B \\
B^{\mathrm{T}} X A & B^{\mathrm{T}} X B
\end{array}\right] .
$$

Lemma 3.4 ([12, p.322], see e.g. [2] for a detailed proof). For any $X=X^{T} \in \mathbb{R}^{n \times n}$, there holds

$\Phi(z)=\left[B^{T}\left(z^{-1} I_{n}-A^{T}\right)^{-1} I_{n}\right] \Pi_{X}\left[\begin{array}{c}\left(z I_{n}-A\right)^{-1} B \\ I_{n}\end{array}\right]$.

Theorem 3.1 Let $r$ denote the normal rank of the spectrum $\Phi(z) .{ }^{1}$ If $X$ is a solution of $C G D A R E(\Sigma)$, the rank of $R_{X}$ is equal to $r$. If $X$ is a solution of DRLMI $(\Sigma)$, the rank of $R_{X}$ is at most equal to $r$.

Proof: Consider $X=X^{\mathrm{T}}$ such that $\Pi_{X} \geq 0$. By Lemma 2.1, in particular (i) $R_{X}$ is positive semidefinite, (ii) $\operatorname{ker} S_{X} \supseteq$ $\operatorname{ker} R_{X}$, and (iii) $Q_{X}-S_{X} R_{X}^{\dagger} S_{X}^{\mathrm{T}}$ is positive semidefinite. Note that (iii) means that $X$ satisfies the Riccati inequality

$$
\begin{aligned}
& \mathscr{D}(X) \stackrel{\text { def }}{=} A^{\mathrm{T}} X A \\
& \quad-\left(A^{\mathrm{T}} X B+S\right)\left(R+B^{\mathrm{T}} X B\right)^{\dagger}\left(B^{\mathrm{T}} X A+S^{\mathrm{T}}\right)+Q-X \geq 0 .
\end{aligned}
$$

Therefore, we can write $\mathscr{D}(X)=H_{X}^{\mathrm{T}} H_{X}$ for some matrix $H_{X}$, which leads to the expression

$$
\Pi_{X}=\left[\begin{array}{c}
S_{X} R_{X}^{\dagger} \\
I
\end{array}\right] R_{X}\left[R_{X}^{\dagger} S_{X}^{\mathrm{T}} I\right]+\left[\begin{array}{c}
H_{X}^{\mathrm{T}} \\
O
\end{array}\right]\left[\begin{array}{ll}
H_{X} & O
\end{array}\right] .
$$

Plugging (23) into (22), $\Phi(z)=W^{\sim}(z) W(z)$ becomes

$\Phi(z)=W_{1}^{\sim}(z) W_{1}(z)+W_{2}^{\sim}(z) W_{2}(z)=\left[W_{1}^{\sim}(z) W_{2}^{\sim}(z)\right]\left[\begin{array}{l}W_{1}(z) \\ W_{2}(z)\end{array}\right]$

where $W_{1}(z)$ is given by

$W_{1}(z)=R_{X}^{\frac{1}{2}}\left[R_{X}^{\dagger} S_{X}^{\mathrm{T}} I\right]\left[\begin{array}{c}\left(z I_{n}-A\right)^{-1} B \\ I_{n}\end{array}\right]$

\footnotetext{
1 The normal rank of a rational matrix $M(z)$ is defined as normrank $M(z) \stackrel{\text { def }}{=} \max _{z \in \mathbb{C}} \operatorname{rank} M(z)$.
}

i.e., $W_{1}(z)=R_{X}^{\frac{1}{2}}\left(R_{X}^{\dagger} S_{X}^{\mathrm{T}}\left(z I_{n}-A\right)^{-1} B+I_{m}\right)$, and $W_{2}(z)$ is

$W_{2}(z)=\left[\begin{array}{ll}H_{X} & O\end{array}\right]\left[\begin{array}{c}\left(z I_{n}-A\right)^{-1} B \\ I_{n}\end{array}\right]=H_{X}\left(z I_{n}-A\right)^{-1} B$.

Notice that $W_{1}(z)=R_{X}^{\frac{1}{2}} T_{X}(z)$, where $T_{X}(z) \stackrel{\text { def }}{=} R_{X}^{\dagger} S_{X}^{\mathrm{T}}\left(z I_{n}-\right.$ $A)^{-1} B+I_{m}$ is square and invertible for all but finitely many $z \in \mathbb{C}$. Its inverse can be written as $T_{X}^{-1}(z)=I_{m}-R_{X}^{\dagger} S_{X}^{\mathrm{T}}\left(z I_{n}-\right.$ $\left.A_{X}\right)^{-1} B$. Thus, the normal rank of

$T_{X}^{-\sim}(z) \Phi(z) T_{X}^{-1}(z)=\left[\begin{array}{ll}R_{X}^{\frac{1}{2}} & T_{X}^{-\sim}(z) W_{2}^{\sim}(z)\end{array}\right]\left[\begin{array}{c}R_{X}^{\frac{1}{2}} \\ W_{2}(z) T_{X}^{-1}(z)\end{array}\right]$

is equal to the normal rank $r$ of $\Phi(z)$. Then, the rank of $R_{X}^{\frac{1}{2}}$, which equals that of $R_{X}$, is not greater than $r$. Now consider the case where $X=X^{\mathrm{T}}$ is a solution of $\operatorname{CGDARE}(\Sigma)$. In this case, the term $H_{X}$ in (23) is zero, and therefore so is the rational function $W_{2}(z)$. As such, $W_{1}(z)$ is a square spectral factor of $\Phi(z)$, i.e., $W^{\sim}(z) W(z)=W_{1}^{\sim}(z) W_{1}(z)$. Moreover, $T_{X}^{-\sim}(z) \Phi(z) T_{X}^{-1}(z)=R_{X}$, which implies that when $X=X^{\mathrm{T}}$ is a solution of CGDARE $(\Sigma)$, the rank of $R_{X}$ is exactly $r$.

Remark 3.1 Theorem 3.1 is strictly related to Theorem 2.4 in [12]. The latter has been derived in the very general setting in which the Popov matrix may not be positive semidefinite. In that case, $\operatorname{rank} R_{X}=r$ for any solution $X$ of CGDARE. Since we are assuming (2), a stronger result holds. Namely, $\operatorname{rank} R_{X} \leq r$ for any solution $X$ of the linear matrix inequality.

\section{Geometric properties of the solutions of GDARE}

Now we show that, given a solution $X$ of $\operatorname{GDARE}(\Sigma)$

(a) $\operatorname{ker} X$ is an output-nulling subspace for the quadruple $(A, B, C, D)$, i.e., $\left[\begin{array}{l}A \\ C\end{array}\right] \operatorname{ker} X \subseteq\left(\operatorname{ker} X \oplus 0_{p}\right)+\operatorname{im}\left[\begin{array}{l}B \\ D\end{array}\right]$;

(b) the gain $K_{X}$ is such that $-K_{X}$ is a friend of $\operatorname{ker} X$, i.e., $\left[\begin{array}{c}A-B K_{X} \\ C-D K_{K}\end{array}\right] \operatorname{ker} X \subseteq \operatorname{ker} X \oplus 0_{p}$.

In the case where $X=X^{\mathrm{T}}$ is the solution of $\operatorname{GDARE}(\Sigma)$ corresponding to the optimal cost, these properties are intuitive. Now we prove that the following stronger result holds.

Proposition 4.1 Let $X$ be the minimal positive semidefinite solution of $\operatorname{GDARE}(\Sigma)$. Then $\operatorname{ker} X$ is the largest outputnulling subspace of the quadruple $(A, B, C, D)$. Moreover, $-K_{X}$ is the corresponding friend.

Proof: Let $x_{0} \in \operatorname{ker} X$. Since the corresponding optimal cost is $J=x_{0}^{\mathrm{T}} X x_{0}=0$, the initial state $x_{0}$ must belong to the largest output-nulling subspace of the quadruple $(A, B, C, D)$. Vice-versa, if we take $x_{0}$ on the largest output-nulling subspace $\mathscr{V}^{\star}$ of $(A, B, C, D)$, we can find $u_{k}(k \geq 0)$ such that 
the state lies on $\mathscr{V}^{\star}$ by maintaining the output at zero, so the corresponding value of the cost is zero. Hence, $x_{0}^{\mathrm{T}} X x_{0}=0$ implies $x_{0} \in \operatorname{ker} X$. The fact that $-K_{X}$ is a friend of $\operatorname{ker} X$ follows from the fact that if $x_{0} \in \operatorname{ker} X$ and we assume by contradiction that $\left(A-B K_{X}\right) x_{0} \notin \operatorname{ker} X$, the corresponding trajectory is not optimal because it is associated with a strictly positive cost. Moreover, since the optimal cost is zero, we must have $\left(C-D K_{X}\right) \operatorname{ker} X=0_{p}$.

Our aim is to prove that (a) and (b) hold for any symmetric solution $X$ of $\operatorname{GDARE}(\Sigma)$.

Theorem 4.1 Let $X$ be a solution of $G D A R E(\Sigma)$. Then, $\operatorname{ker} X$ is an output-nulling subspace of the quadruple $(A, B, C, D)$ and $-K_{X}$ is a friend of $\operatorname{ker} X$.

Proof: Since $X$ is a solution of $\operatorname{GDARE}(\Sigma)$, the identity

$$
X=A_{X}^{\mathrm{T}} X A_{X}+Q_{0 X}
$$

holds, where $Q_{0 X} \stackrel{\text { def }}{=}\left[\begin{array}{ll}I_{n} & -S_{X} R_{X}^{\dagger}\end{array}\right]\left[\begin{array}{cc}Q & S \\ S^{\mathrm{T}} & R\end{array}\right]\left[\begin{array}{c}I_{n} \\ -R_{X}^{\dagger} S_{X}^{\mathrm{T}}\end{array}\right] \geq 0$. From Lemma 3.1, $\operatorname{ker} X$ is $A_{X}$-invariant and is contained in $\operatorname{ker} Q_{0 X}$. By factorising $\Pi$ as in (21), we get $Q_{0 X}=C_{X}^{\mathrm{T}} C_{X}$ where

$$
C_{X} \stackrel{\text { def }}{=}\left[\begin{array}{ll}
C & D
\end{array}\right]\left[\begin{array}{c}
I_{n} \\
-R_{X}^{\dagger} S_{X}^{\mathrm{T}}
\end{array}\right]=C-D R_{X}^{\dagger} S_{X}^{\mathrm{T}} .
$$

Hence, $\operatorname{ker} X$ is also contained in $\operatorname{ker} C_{X}$ so that $\operatorname{ker} X$ is output-nulling and $-K_{X}$ is a friend of $\operatorname{ker} X$.

Now we provide a characterisation of the reachable subspace on $\operatorname{ker} X$. We show that this subspace plays a crucial role in the solution of the associated optimal control problem. We recall that the reachable subspace $\mathscr{R}_{\mathscr{V}}^{\star}$ on an outputnulling subspace $\mathscr{V}$ is the subspace of points of $\mathscr{V}$ that can be reached from the origin along trajectories contained on $\mathscr{V}$ by at the same time maintaining the output at zero.

We will show that the reachable subspace $\mathscr{R}_{\operatorname{ker} X}^{\star}$ on $\operatorname{ker} X$, coincides with the reachable subspace of the pair $\left(A_{X}, B G_{X}\right)$. To prove this fact, we first need to give some additional results on the solutions of $\operatorname{CGDARE}(\Sigma)$. In particular, we now focus our attention on the term $R_{X}$. Clearly, when $X$ is positive semidefinite, the null-space of $R_{X}$ is the intersection of the null-space of $R$ with that of $X B$. This result, which is intuitive and easy to prove for positive semidefinite solutions of $\operatorname{CGDARE}(\Sigma)$, holds for any solution. However, in this case the proof - which is divided between Lemma 4.1 and Lemma 4.2 presented below - is more involved, and requires the machinery constructed in the first part of the paper.

Lemma 4.1 Let $X=X^{T}$ be a solution of $C G D A R E(\Sigma), C_{X}$ be defined by (25) and

$$
\mathscr{R}_{0} \stackrel{\text { def }}{=} \mathrm{im}\left[\begin{array}{lllll}
B G_{X} & A_{X} B G_{X} & A_{X}^{2} B G_{X} & \ldots & A_{X}^{n-1} B G_{X}
\end{array}\right] .
$$

Then,

$$
\operatorname{ker} R_{X} \subseteq \operatorname{ker} R, \quad \text { and } \quad \mathscr{R}_{0} \subseteq \operatorname{ker} C_{X}
$$

Proof: Since the columns of $G_{X}$ span $\operatorname{ker} R_{X}$, we need to show that $R G_{X}=0$. Recall from the proof of Theorem 3.1 that when $X=X^{\mathrm{T}}$ is a solution of $\operatorname{CGDARE}(\Sigma)$, $\Phi(z)$ can be written as $\Phi(z)=W^{\sim}(z) W(z)=T_{X}^{\sim}(z) R_{X} T_{X}(z)$ where $T_{X}(z)=R_{X}^{\dagger} S_{X}^{\mathrm{T}}\left(z I_{n}-A\right)^{-1} B+I_{m}$ is square and invertible for all but finitely many $z \in \mathbb{C}$. Hence, we have $R_{X}=\left[W(z) T_{X}^{-1}(z)\right]^{\sim}\left[W(z) T_{X}^{-1}(z)\right]$ so that $R_{X} G_{X}=0 \mathrm{im}-$ plies $W(z) T_{X}^{-1}(z) G_{X} \equiv 0$. Recall from the proof of Theorem 3.1 that $T_{X}^{-1}(z)=I_{m}-R_{X}^{\dagger} S_{X}^{\mathrm{T}}\left(z I_{n}-A_{X}\right)^{-1} B$, so that

$W(z) T_{X}^{-1}(z)=\left(C\left(z I_{n}-A\right)^{-1} B+D\right)\left(I_{m}-R_{X}^{\dagger} S_{X}^{\mathrm{T}}\left(z I_{n}-A_{X}\right)^{-1} B\right)$.

Consider the following term of the product:

$$
H(z)=C\left(z I_{n}-A\right)^{-1} B R_{X}^{\dagger} S_{X}^{\mathrm{T}}\left(z I_{n}-A_{X}\right)^{-1} B .
$$

By noticing that $B R_{X}^{\dagger} S_{X}^{\mathrm{T}}=A-A_{X}=\left(z I_{n}-A_{X}\right)-\left(z I_{n}-A\right)$, we obtain $H(z)=C\left(z I_{n}-A\right)^{-1} B-C\left(z I_{n}-A_{X}\right)^{-1} B$. Hence,

$$
\begin{aligned}
W(z) T_{X}^{-1}(z) & =W(z)-D R_{X}^{\dagger} S_{X}^{\mathrm{T}}\left(z I_{n}-A_{X}\right)^{-1} B-H(z) \\
& =D+C_{X}\left(z I_{n}-A_{X}\right)^{-1} B .
\end{aligned}
$$

Since $W(z) T_{X}^{-1}(z) G_{X}$ is identically zero, it must be zero also when $z \rightarrow \infty$. In particular, $D G_{X}=0$, so that $R G_{X}=0$, which yields the first of (27). From $W(z) T_{X}^{-1}(z) G_{X} \equiv 0$ we also get $C_{X}\left(z I-A_{X}\right)^{-1} B G_{X} \equiv 0$ so that the reachable subspace of the pair $\left(A_{X}, B G_{X}\right)$, i.e. (26), is contained in $\operatorname{ker} C_{X}$ so that also the second of (27) holds.

In Lemma 4.1 we have shown that $\operatorname{ker} R_{X} \subseteq \operatorname{ker} R$. Since $R_{X}=R+B^{\mathrm{T}} X B$, it also straightforwardly follows that $\operatorname{ker} R_{X} \subseteq \operatorname{ker}\left(B^{\mathrm{T}} X B\right)$ for any solution $X$ of $\operatorname{CGDARE}(\Sigma)$. However, a stronger result holds, which says that $\operatorname{ker} R_{X} \subseteq \operatorname{ker}(X B)$. This is an obvious consequence of Lemma 4.1 for any solution $X \geq 0$, while it is a quite surprising and deep geometric result in the general case.

Lemma 4.2 Let $X=X^{T}$ be a solution of $C G D A R E(\Sigma)$. Then,

$$
\operatorname{ker} R_{X} \subseteq \operatorname{ker}(X B)
$$

Proof: From Lemma 4.1, if $v \in \operatorname{ker} R_{X}$, then $v \in \operatorname{ker} R \cap$ $\operatorname{ker}\left(B^{\mathrm{T}} X B\right)$. We can select a change of coordinates in the input space $\mathbb{R}^{m}$ induced by the $m \times m$ orthogonal matrix $T_{X}=$ $\left[\begin{array}{ll}T_{1 X} & T_{2 X}\end{array}\right]$ where $\operatorname{im} T_{1 X}=\operatorname{im} R_{X}$ and $\operatorname{im} T_{2 X}=\operatorname{im} G_{X}=$ $\operatorname{ker} R_{X}$. In this basis $R_{X}$ is block-diagonal, with the first block being non-singular and the second being zero. Since ker $R \supseteq$ $\operatorname{ker} R_{X}$ as proved in Lemma 4.1, matrix $R$ in this basis has the form $R=\left[\begin{array}{rr}R_{1} & 0 \\ 0 & 0\end{array}\right]$. In the same basis, matrix $B$ can be partitioned accordingly as $B=\left[\begin{array}{ll}B_{1} & B_{2}\end{array}\right]$, so that $\operatorname{im} B_{2}=$ $\operatorname{im}\left(B G_{X}\right)$. We must show that $X B_{2}=0$. Since $\operatorname{ker} R_{X} \subseteq$ 
$\operatorname{ker}\left(B^{\mathrm{T}} X B\right)$, in this basis we find

$$
B^{\mathrm{T}} X B_{2}=\left[\begin{array}{c}
B_{1}^{\mathrm{T}} \\
B_{2}^{\mathrm{T}}
\end{array}\right] X B_{2}=0
$$

Moreover, since $\operatorname{ker} R \subseteq \operatorname{ker} S$, in the selected basis $S$ takes the form $S=\left[\begin{array}{ll}S_{1} & 0\end{array}\right]$. Thus, $S_{X}=A^{\mathrm{T}} X B+S=$ $\left[A^{\mathrm{T}} X B_{1}+S_{1} A^{\mathrm{T}} X B_{2}\right]$. From $\operatorname{ker} R_{X} \subseteq \operatorname{ker} S_{X}$ it now follows that $A^{\mathrm{T}} X B_{2}=0$ which, together with (29), yields

$$
\left[\begin{array}{l}
A^{\mathrm{T}} \\
B^{\mathrm{T}}
\end{array}\right] X B_{2}=0
$$

If $A$ is non-singular or, more in general, if the zero eigenvalue of $A$, when present, is controllable from $B$, then clearly $X B_{2}=0$. However, this result is true in general, without any assumption. To prove this, let us consider $\mathscr{R}_{0}$ defined in (26) which, in the chosen input space basis, is the reachable subspace of the pair $\left(A_{X}, B_{2}\right){ }^{2}$ Let us consider a basis of the state-space where the pair $\left(A_{X}, B_{2}\right)$ are in Kalman controllability form. In such a basis, the subspace $\mathscr{R}_{0}$ is spanned by the columns of the matrix $\left[\begin{array}{l}I \\ O\end{array}\right]$ and we have

$A_{X}=\left[\begin{array}{cc}A_{X, 11} & A_{X, 12} \\ O & A_{X, 22}\end{array}\right], \quad B_{2}=\left[\begin{array}{c}B_{21} \\ O\end{array}\right], \quad B_{1}=\left[\begin{array}{c}B_{11} \\ B_{12}\end{array}\right]$,

where $\left(A_{X, 11}, B_{21}\right)$ is reachable. In this basis, $C_{X}=\left[O \mid C_{X, 1}\right]$ in view of the second of (27). Since $A_{X}=A-B K_{X}$, we can re-write (30) as $\left[\begin{array}{c}A_{X}^{\mathrm{T}}+K_{X}^{\mathrm{T}} B^{\mathrm{T}} \\ B^{\mathrm{T}}\end{array}\right] X B_{2}=0$ or, equivalently, as $\left[\begin{array}{c}A_{X}^{\mathrm{T}} \\ B^{\mathrm{T}}\end{array}\right] X B_{2}=0$. We can write this equation as

$$
\left[\begin{array}{cc}
A_{X, 11}^{\mathrm{T}} & O \\
A_{X, 12}^{\mathrm{T}} & A_{X, 22}^{\mathrm{T}} \\
B_{11}^{\mathrm{T}} & B_{12}^{\mathrm{T}} \\
B_{21}^{\mathrm{T}} & O
\end{array}\right]\left[\begin{array}{ll}
X_{11} & X_{12} \\
X_{12}^{\mathrm{T}} & X_{22}
\end{array}\right]\left[\begin{array}{c}
B_{21} \\
O
\end{array}\right]=0
$$

We want to show that $\left[\begin{array}{ll}X_{11} & X_{12} \\ X_{12}^{\mathrm{T}} & X_{22}\end{array}\right]\left[\begin{array}{c}B_{21} \\ O\end{array}\right]=0$, i.e., that $X_{11} B_{21}=$ 0 and $X_{12}^{\mathrm{T}} B_{21}=0$. From (32) we find

$$
\begin{aligned}
A_{X, 11}^{\mathrm{T}} X_{11} B_{21} & =0, \\
B_{21}^{\mathrm{T}} X_{11} B_{21} & =0 .
\end{aligned}
$$

Since the pair $\left(A_{X, 11}, B_{21}\right)$ is reachable by construction, $X_{11} B_{21}=0$. It remains to show that $X_{12}^{\mathrm{T}} B_{21}=0$. In this basis,

\footnotetext{
2 In the symbol denoting this subspace we dropped the subscript $X$ because, as it will be proved in the sequel, this subspace is independent of the particular solution of the $\operatorname{CGDARE}(\Sigma)$.
}

equation (24) - which is exactly $\operatorname{GDARE}(\Sigma)$ - now reads as

$$
\left[\begin{array}{ll}
X_{11} & X_{12} \\
X_{12}^{\mathrm{T}} & X_{22}
\end{array}\right]=\left[\begin{array}{cc}
A_{X, 11}^{\mathrm{T}} & O \\
A_{X, 12}^{\mathrm{T}} & A_{X, 22}^{\mathrm{T}}
\end{array}\right]\left[\begin{array}{l}
X_{11} X_{12} \\
X_{12}^{\mathrm{T}} X_{22}
\end{array}\right]\left[\begin{array}{cc}
A_{X, 11} & A_{X, 12} \\
O & A_{X, 22}
\end{array}\right]+\left[\begin{array}{cc}
O & O \\
O & C_{X, 1}^{\mathrm{T}} C_{X, 1}
\end{array}\right]
$$

from which we find in particular $X_{11}=A_{X, 11}^{\mathrm{T}} X_{11} A_{X, 11}$. This equation can be written together with (33) and (34) as

$$
\left[\begin{array}{cc}
A_{X, 11}^{\mathrm{T}} X_{11} A_{X, 11}-X_{11} & A_{X, 11}^{\mathrm{T}} X_{11} B_{21} \\
B_{21}^{\mathrm{T}} X_{11} A_{X, 11} & B_{21}^{\mathrm{T}} X_{11} B_{21}
\end{array}\right]=0 .
$$

Since the pair $\left(A_{X, 11}, B_{21}\right)$ is reachable, we can apply Lemma 2.9 in [12], which guarantees that $X_{11}$ is zero. Now we can re-write (24) as

$$
\left[\begin{array}{cc}
O & X_{12} \\
X_{12}^{\mathrm{T}} & X_{22}
\end{array}\right]=\left[\begin{array}{cc}
A_{X, 11}^{\mathrm{T}} & O \\
A_{X, 12}^{\mathrm{T}} & A_{X, 22}^{\mathrm{T}}
\end{array}\right]\left[\begin{array}{cc}
O & X_{12} \\
X_{12}^{\mathrm{T}} & X_{22}
\end{array}\right]\left[\begin{array}{cc}
A_{X, 11} & A_{X, 12} \\
O & A_{X, 22}
\end{array}\right]+\left[\begin{array}{lc}
O & O \\
O & C_{X, 1}^{\mathrm{T}} C_{X, 1}
\end{array}\right]
$$

In particular, we get

$$
X_{12}=A_{X, 11}^{\mathrm{T}} X_{12} A_{X, 22}
$$

Plugging $X_{11}=0$ into (32), we obtain

$$
B_{21}^{\mathrm{T}} X_{12} A_{X, 22}=0
$$

Equations (35) and (36) can be re-written as

$$
\left[\begin{array}{c}
A_{X, 11}^{\mathrm{T}} \\
B_{21}^{\mathrm{T}}
\end{array}\right] X_{12} A_{X, 22}=\left[\begin{array}{c}
X_{12} \\
O
\end{array}\right]
$$

Applying Lemma 3.3 for $k=0$, we get $B_{21}^{\mathrm{T}} X_{12}=0$.

Remark 4.1 In the last line of the proof of Lemma 4.2 we can apply Lemma 3.3 for $k \geq 0$, and obtain $X_{12}^{\mathrm{T}} A_{X, 11}^{k} B_{21}=0$ for all $k \geq 0$. Since the pair $\left(A_{X, 11}, B_{21}\right)$ is reachable, this yields $X_{12}=0$. Therefore the following stronger result holds.

Proposition 4.2 Let $X=X^{T}$ be a solution of $C G D A R E(\Sigma)$ and $\mathscr{R}_{0}$ be defined by (26). Then, $X \mathscr{R}_{0}=0_{n}$.

Remark 4.2 As an obvious corollary of Lemmas 4.1 and 4.2, we have $\operatorname{ker} R_{X}=\operatorname{ker}(X B) \cap \operatorname{ker} R=\operatorname{ker}\left[\begin{array}{c}X B \\ R\end{array}\right]$.

Remark 4.3 The result established in Lemma 4.2 does not continue to hold if we only assume that $X=X^{\mathrm{T}}$ is a solution of $\operatorname{GDARE}(\Sigma)$. Consider e.g. $A=\left[\begin{array}{cc}-1 & 0 \\ -5 & -6\end{array}\right], B=\left[\begin{array}{cc}-4 & 0 \\ 0 & -2\end{array}\right]$, $C=\left[\begin{array}{ll}0 & 1\end{array}\right]$ and $D=\left[\begin{array}{ll}4 & 0\end{array}\right]$. It can be easily verified that $X=$ $\operatorname{diag}\{-1,1\}$ is a solution of the $\operatorname{GDARE}(\Sigma)$ but not of the $\operatorname{CGDARE}(\Sigma)$. Here $\operatorname{ker} R_{X}=\operatorname{im}\left[\begin{array}{l}1 \\ 0\end{array}\right] \neq \operatorname{ker}\left[\begin{array}{c}X B \\ R\end{array}\right]=0_{m}$. 
Remark 4.4 The result established in Lemma 4.2 does not hold when $\Pi$ is not positive semidefinite. Consider e.g. $A=\left[\begin{array}{ll}1 & 1 \\ 1 & 1\end{array}\right], B=\left[\begin{array}{ll}0 & 1 \\ 0 & 1\end{array}\right], Q=\left[\begin{array}{cc}1 & 0 \\ 0 & -1\end{array}\right], S=\left[\begin{array}{ll}0 & 0 \\ 0 & 0\end{array}\right]$ and $R=\left[\begin{array}{ll}1 & 0 \\ 0 & 0\end{array}\right]$. A solution of $\operatorname{CGDARE}(\Sigma)$ is given by $X=\operatorname{diag}\{1,-1\}$. Indeed, $X$ satisfies (4) since $S_{X}=0$ and $\operatorname{ker} R_{X}=\operatorname{im}\left[\begin{array}{l}0 \\ 1\end{array}\right]$. Changing coordinates in the input space as shown in the proof of Lemma 4.2 leads to $B_{1}=\left[\begin{array}{l}0 \\ 0\end{array}\right]$ and $B_{2}=\left[\begin{array}{l}1 \\ 1\end{array}\right]$. However, this time $X B_{2}=\left[\begin{array}{c}1 \\ -1\end{array}\right]$.

Theorem 4.2 Let $X=X^{T}$ be a solution of CGDARE( $(\Sigma)$. Let $\mathscr{R}_{0}$ denote the reachable subspace of the pair $\left(A_{X}, B G_{X}\right)$ as defined in (26), and $\mathscr{R}_{\mathrm{ker} X}^{\star}$ be the largest reachability subspace on the output-nulling subspace $\operatorname{ker} X$. Then,

$$
\mathscr{R}_{\text {ker } X}^{\star}=\mathscr{R}_{0} .
$$

Proof: Let us first show that

$$
\operatorname{im}\left(B G_{X}\right)=\operatorname{ker} X \cap B \operatorname{ker} D .
$$

We recall that $\operatorname{im} G_{X}=\operatorname{ker} R_{X}$. From Remark 4.2 we know that $\operatorname{ker} R_{X}=\operatorname{ker}(X B) \cap \operatorname{ker} R$. Then $\operatorname{im}\left(B G_{X}\right)=B \operatorname{ker} R_{X}=$ $B(\operatorname{ker}(X B) \cap \operatorname{ker} R)=\operatorname{ker} X \cap B \operatorname{ker} R=\operatorname{ker} X \cap B \operatorname{ker} D$. Subspace $\mathscr{R}_{0}$ is by definition the smallest $A_{X}$-invariant subspace containing $\operatorname{im}\left(B G_{X}\right)=\operatorname{ker} X \cap B \operatorname{ker} D$. On the other hand, $\mathscr{R}_{\mathrm{ker} X}^{\star}$ is characterised as follows $[9, \mathrm{p} .424]$ : Let $F$ be an arbitrary friend of $\operatorname{ker} X$, i.e., $F$ is any feedback matrix such that $(A+B F) \operatorname{ker} X \subseteq \operatorname{ker} X$ and $(C+D F) \operatorname{ker} X=0_{p}$. Then $\mathscr{R}_{\mathrm{ker} X}^{\star}$ is the smallest $(A+B F)$-invariant subspace containing $\operatorname{ker} X \cap B \operatorname{ker} D$. Note that $\mathscr{R}_{\operatorname{ker} X}^{\star}$ does not depend on the choice of the friend $F$, [13, Theorem 7.18]. We have seen in Theorem 4.1 that $F=-K_{X}$ is a particular friend of $\operatorname{ker} X$. For this choice of $F$, we have $A+B F=A-B K_{X}=A_{X}$, so that $\mathscr{R}_{\mathrm{ker} X}^{\star}$ is the smallest $A_{X}$-invariant subspace containing $\operatorname{ker} X \cap B \operatorname{ker} D$, which is the definition of $\mathscr{R}_{0}$.

In [12] it is proved that the inertia of $R_{X}$ is independent of the solution $X=X^{\mathrm{T}}$ of $\operatorname{CGDARE}(\Sigma)$. Here, we show that much more is true when $\Pi \geq 0$, i.e., $\operatorname{ker} R_{X}$ is independent of the solution $X=X^{\mathrm{T}}$ of $\operatorname{CGDARE}(\Sigma)$.

Theorem 4.3 Let $X_{1}, X_{2}$ be two solutions of CGDARE $(\Sigma)$. Then, $\operatorname{ker} R_{X_{1}}=\operatorname{ker} R_{X_{2}}$.

Proof: Consider two solutions $X_{1}=X_{1}^{\mathrm{T}}$ and $X_{2}=X_{2}^{\mathrm{T}}$ of $\operatorname{CGDARE}(\Sigma)$. In particular, $X_{1}$ and $X_{2}$ also satisfy the generalised Riccati inequality, so that $\Pi_{X_{1}} \geq 0$ and $\Pi_{X_{2}} \geq 0$. In other words, with the same notation used in the proof of Theorem 3.1, $\Pi_{X_{i}}=L\left(X_{i}\right)+\Pi \geq 0$ for $i \in\{1,2\}$. The set of solutions of the generalised Riccati inequality is a convex set, i.e., by taking $\alpha \in(0,1)$, then $\alpha \Pi_{X_{1}}+(1-\alpha) \Pi_{X_{2}}$ is positive semidefinite because it is a convex combination of positive semidefinite terms. For our purposes, it is sufficient to fix an arbitrary value of $\alpha$, say $\alpha=\frac{1}{2}$. Then,

$0 \leq \frac{1}{2}\left(\Pi_{X_{1}}+\Pi_{X_{2}}\right)=\Pi_{\frac{1}{2}\left(X_{1}+X_{2}\right)}=\Pi+L\left(\frac{1}{2}\left(X_{1}+X_{2}\right)\right)$,

where the last equality holds in view of the linearity of $L(\cdot)$. This means that $Y \stackrel{\text { def }}{=} \frac{1}{2}\left(X_{1}+X_{2}\right)$ satisfies the Riccati inequality $\Pi_{Y} \geq 0$. By virtue of Theorem 3.1, the rank of $R_{Y} \stackrel{\text { def }}{=} R+B^{\mathrm{T}} Y B$ is not greater than $r$. On the other hand,

$$
R_{Y}=R+\frac{1}{2} B^{\mathrm{T}}\left(X_{1}+X_{2}\right) B=\frac{1}{2}\left(R_{X_{1}}+R_{X_{2}}\right) .
$$

Hence, since $X_{1}$ and $X_{2}$ are both solutions of CGDARE $(\Sigma)$, the ranks of $R_{X_{1}}$ and $R_{X_{2}}$ are exactly equal to $r$. Thus, the rank of $R_{Y}$ is greater or equal to $r$. This means that the rank of $R_{Y}$ must be exactly equal to $r$, i.e., from (40) we have that $R_{X_{1}}$ and $R_{X_{2}}$ must have the same null-space.

Now we want to prove that the subspace $\mathscr{R}_{\mathrm{ker} X}^{\star}$ is independent of the particular solution $X=X^{\mathrm{T}}$ of $\operatorname{CGDARE}(\Sigma)$. Moreover, $A_{X}$ restricted to this subspace does not depend on the particular solution $X=X^{\mathrm{T}}$ of $\operatorname{CGDARE}(\Sigma)$.

Theorem 4.4 Let $X$ and $Y$ be two solutions of CGDARE $(\Sigma)$. Let $A_{X}$ and $A_{Y}$ be the corresponding closed-loop matrices. Then, $\mathscr{R}_{\mathrm{ker} X}^{\star}=\mathscr{R}_{\mathrm{ker} Y}^{\star}$, and $\left.A_{X}\right|_{\mathscr{R}_{\mathrm{ker} X}^{\star}}=\left.A_{Y}\right|_{\mathscr{R}_{\mathrm{ker} Y}^{\star}}$.

Proof: Let $\Delta \stackrel{\text { def }}{=} Y-X$. Since $\operatorname{ker} R_{X}$ coincides with $\operatorname{ker} R_{Y}$ by virtue of Theorem 4.3 , we have $R_{X}^{\dagger}=R_{Y}^{\dagger} R_{Y} R_{X}^{\dagger}$ so that

$A_{X}-A_{Y}=B\left(R_{Y}^{\dagger} S_{Y}^{\mathrm{T}}-R_{X}^{\dagger} S_{X}^{\mathrm{T}}\right)=B R_{Y}^{\dagger}\left(S_{Y}^{\mathrm{T}}-R_{Y} R_{X}^{\dagger} S_{X}^{\mathrm{T}}\right)$.

Plugging $S_{Y}^{\mathrm{T}}=B^{\mathrm{T}} Y A+S^{\mathrm{T}}=B^{\mathrm{T}} \Delta A+S_{X}^{\mathrm{T}}$ and $R_{Y}=R_{X}+B^{\mathrm{T}} \Delta B$ into (41) yields $A_{X}-A_{Y}=B R_{Y}^{\dagger}\left(B^{\mathrm{T}} \Delta A-B^{\mathrm{T}} \Delta B R_{X}^{\dagger} S_{X}^{\mathrm{T}}\right)=$ $B R_{Y}^{\dagger} B^{\mathrm{T}} \Delta A_{X}$. This means that $A_{Y}=A_{X}-B R_{Y}^{\dagger} B^{\mathrm{T}} \Delta A_{X}$. We already know that in a suitable basis of the state space such that the first coordinates span $\mathscr{R}_{\operatorname{ker} X}^{\star}$ and a suitable orthogonal basis of the input space such that the second group of coordinates span $\operatorname{ker} R_{X}$, matrices $A_{X}$ and $B$ can be written as in (31), see Lemma 4.2. The reachable subspace of the pair $\left(A_{X}, B G_{X}\right)$ is written in this basis as $\mathscr{R}_{0}=\left[\begin{array}{l}I \\ o\end{array}\right]$. We want to show that, in the same basis, we also have

$$
A_{Y}=\left[\begin{array}{cc}
A_{X, 11} & A_{Y, 12} \\
O & A_{Y, 22}
\end{array}\right], \quad \text { and } \quad B_{2}=\left[\begin{array}{c}
B_{21} \\
O
\end{array}\right]
$$

In fact, if this is the case, the reachable subspace of the pair $\left(A_{Y}, B G_{Y}\right)$ exactly coincides with the reachable subspace of the pair $\left(A_{X}, B G_{X}\right)$, i.e., with $\mathscr{R}_{0}$, because the pair $\left(A_{Y, 22}, 0\right)$ is completely non controllable. In the chosen basis, the difference $\Delta=Y-X$ can be written as $\Delta=\operatorname{diag}\left\{O, \Delta_{2}\right\}$ in view 
of [12, Theorem 2.10]. ${ }^{3}$ Thus

$A_{Y}=A_{X}-B R_{Y}^{\dagger} B^{\mathrm{T}}\left[\begin{array}{cc}O & O \\ O & \Delta_{2}\end{array}\right]\left[\begin{array}{cc}A_{X, 11} & A_{X, 12} \\ O & A_{X, 22}\end{array}\right]=\left[\begin{array}{cc}A_{X, 11} \star \\ O & \star\end{array}\right]$.

Therefore, the reachable subspace of the pair $\left(A_{Y}, B G_{Y}\right)$ is exactly $\mathscr{R}_{0}=\left[\begin{array}{l}I \\ o\end{array}\right]$, and $\left.A_{X}\right|_{\mathscr{R}_{\mathrm{ker} X}^{\star}}=\left.A_{Y}\right|_{\mathscr{R}_{\mathrm{ker} Y}^{\star}}=A_{X, 11}$.

\section{Stabilisation}

In the previous sections, we have observed that the eigenvalues of $A_{X}$ restricted to $\mathscr{R}_{0}$ are independent of the solution $X=X^{\mathrm{T}}$ of $\operatorname{CGDARE}(\Sigma)$. This means that these eigenvalues are present in the closed-loop regardless of the solution $X=X^{\mathrm{T}}$ of $\operatorname{CGDARE}(\Sigma)$ that we consider. On the other hand, $\mathscr{R}_{0}$ coincides with the subspace $\mathscr{R}_{\mathrm{ker} X}^{\star}$, which is by definition the smallest $\left(A-B K_{X}\right)$-invariant subspace containing $\operatorname{im}\left(B G_{X}\right)$. Then, we can always find a matrix $L$ that assigns all the eigenvalues of $\left(A_{X}+B G_{X} L\right)$ restricted to $\mathscr{R}_{\mathrm{ker} X}^{\star}$, by adding a further term $B G_{X} L x_{k}$ to the feedback control law, because this does not change the value of the cost with respect to the one obtained by $u_{k}=-K_{X} x_{k}$. In doing so, we can stabilise the closed-loop if $\operatorname{ker} X$ is externally stabilised by $-K_{X}$. We show this fact in the following example.

Example 5.1 Consider a Popov triple in which $A=\left[\begin{array}{ll}1 & 1 \\ 0 & 1\end{array}\right]$, $B=\left[\begin{array}{ll}2 & 0 \\ 1 & 1\end{array}\right], Q=\left[\begin{array}{ll}0 & 0 \\ 0 & 1\end{array}\right], S=\left[\begin{array}{ll}0 & 0 \\ 0 & 0\end{array}\right]$ and $R=\left[\begin{array}{ll}0 & 0 \\ 0 & 0\end{array}\right]$. The matrix $X=\operatorname{diag}\{0,1\}$ is the only solution of $\operatorname{GDARE}(\Sigma)$ but not a solution of DARE $(\Sigma)$, since $R+B^{\mathrm{T}} X B$ is singular. Hence, $\operatorname{DARE}(\Sigma)$ does not admit solutions. The closed-loop matrix is $A_{X}=\operatorname{diag}\{1,0\}$, and the resulting closed-loop system is not asymptotically stable. However, the solution $X$ of $\operatorname{GDARE}(\Sigma)$ is optimal for the LQ problem, because it leads to the cost $J^{*}=x_{2}^{2}(0)$ which cannot be decreased. Now, consider the gain $K=B^{-1} A$, which leads to the closed-loop matrix $A_{\mathrm{CL}}=A-B K=0$, and the value of the performance index associated with this closed-loop is again $J=x_{2}^{2}(0)=J^{*}$. Therefore, this is another optimal solution of the LQ problem, which differently from $X$ is also stabilising. However, this optimal solution is not associated with any solution of $\operatorname{GDARE}(\Sigma)$, since $X$ is the only solution of $\operatorname{GDARE}(\Sigma)$. This example shows that there can be an optimal control which

\footnotetext{
3 The result in [12, Theorem 2.10] is shown in a basis that is the same considered here. Indeed, the basis of the state space considered in [12, Theorem 2.10] has the first coordinates spanning the largest controllability subspace of the quadruple $\left(A, B, S_{X}^{\mathrm{T}}, R_{X}\right)$. However, this subspace coincides with the largest controllability subspace of a quadruple obtained from the previous one by applying the control input $u_{t}=-K_{X} x_{t}+H_{X} v_{t}$, where $\operatorname{im} H_{X}=\operatorname{ker} R_{X}$. The quadruple thus obtained is exactly $\left(A_{X}, B G_{X}, S_{X}^{\mathrm{T}}-R_{X} R_{X}^{\mathrm{T}} S_{X}^{\mathrm{T}}, 0\right)=\left(A_{X}, B G_{X}, 0,0\right)$, and the corresponding largest controllability subspace is indeed $\mathscr{R}_{\operatorname{ker} X}^{\star}$.
}

is stabilising, but no stabilising solutions of $\operatorname{GDARE}(\Sigma)$ exist. This fact can be explained on the basis of the fact that the set of all solutions of the LQ problem is given by $\mathscr{U}_{k}=\left\{-K_{X} x_{k}+G_{X} v_{k} \mid v_{k} \in \mathbb{R}^{m}\right\}$, where $X$ is the optimizing solution of $\operatorname{GDARE}(\Sigma)$ and $G_{X}=\left(I_{m}-R_{X}^{\dagger} R_{X}\right)=\frac{1}{2}\left[\begin{array}{cc}1 & -1 \\ -1 & 1\end{array}\right]$. Therefore, the problem becomes that of using the degree of freedom given by $v_{k}$ to find a closed-loop solution that is optimal and also stabilising, i.e., to determine a matrix $L$ in

$$
x_{t+1}=\left(A-B K_{X}\right) x_{t}+B G_{X} L x_{t}=A_{X} x_{t}+B G_{X} L x_{t}
$$

such that the closed-loop $A_{\mathrm{CL}}=A_{X}+B G_{X} L$ is stabilised. It is easy to see that, in general, the set of all optimal closed loop matrices $A_{\mathrm{CL}}=A_{X}+B G_{X} L$ are parameterised by $A_{\mathrm{CL}}=\left[\begin{array}{ll}\alpha & \beta \\ 0 & 0\end{array}\right]$ where $\alpha$ and $\beta$ can be arbitrarily chosen by selecting a suitable $L$. In fact, since $B G_{X}=\left[\begin{array}{cc}1 & -1 \\ 0 & 0\end{array}\right]$, by choosing $L=\left[\begin{array}{cc}\alpha-1 & \beta \\ 0 & 0\end{array}\right]$ we obtain the desired form for the closed-loop matrix. Hence, in particular, we can obtain a zero or nilpotent closed-loop matrix. In both cases, the cost is the same and is equal to $J^{*}=x_{2}^{2}(0)$.

In other words, there is only one solution to $\operatorname{GDARE}(\Sigma)$ and is not stabilising, and all the optimal solutions of the optimal control problem are given by the closed-loop matrix $A_{X}+$ $B G_{X} L$, where $L$ is a degree of freedom. By using this degree of freedom, we have found solutions of the optimal control problem that are stabilising but which do not correspond to stabilising solutions of $\operatorname{GDARE}(\Sigma)$, because $\operatorname{GDARE}(\Sigma)$ does not have stabilising solutions.

Example 5.2 Consider an infinite-horizon LQ problem with $A=\left[\begin{array}{cc}0 & 0 \\ -7 & -4\end{array}\right], B=\left[\begin{array}{ll}0 & 0 \\ 5 & 9\end{array}\right], C=\left[\begin{array}{ll}-3 & -5\end{array}\right]$, and $D=\left[\begin{array}{ll}0 & -3\end{array}\right]$. Let $Q=C^{\mathrm{T}} C, S=C^{\mathrm{T}} D$ and $R=D^{\mathrm{T}} D$. This system has one invariant zero at the origin. Moreover, it is not leftinvertible. Thus, $\operatorname{DARE}(\Sigma)$ cannot be solved. However, the $2 \times 2$ zero matrix is a solution of $\operatorname{CGDARE}(\Sigma)$. The corresponding closed-loop matrix is $A_{X}=\left[\begin{array}{cc}0 & 0 \\ -16 & -19\end{array}\right]$. The eigenvalue at the origin is fixed because it is an invariant zero of the system. The remaining eigenvalue -19 , which is unstable, is an eigenvalue of $A_{X}$ restricted to $\mathscr{R}_{\operatorname{ker} X}^{\star}$, and it can therefore be placed arbitrarily in the complex plane without affecting the cost. Matrix $G_{X}=I_{m}-R_{X}^{\dagger} R_{X}=\operatorname{diag}\{1,0\}$ is such that $\operatorname{im}\left(B G_{X}\right)=\operatorname{im}\left[\begin{array}{l}0 \\ 1\end{array}\right]$ as expected. By using the standard procedure to assign the eigenvalue $1 / 2$ we obtain

$L=\left[\begin{array}{cc}84 / 273 & 39 / 10 \\ -1 & -5 / 3\end{array}\right]$

It is easy to check that the eigevalues of $A_{X}+B G_{X} L$ are exactly $\{0,0.5\}$. 


\section{Concluding remarks}

In this paper we presented a self-contained analysis of some structural properties of the CGDARE that arises in infinitehorizon discrete LQ optimal control. The considerations that emerged from this analysis revealed that a subspace $\mathscr{R}_{0}$ can be identified that is independent of the particular solution of CGDARE considered. Even more importantly, it has been shown that the closed-loop matrix restricted to this subspace does not depend on the particular solution of CGDARE, and has been shown to be fixed for any state-feedback control constructed from a solution of the CGDARE. On the other hand, if such subspace is not zero, in the optimal control a further term can be added to the state-feedback generated from the solution of the Riccati equation that does not modify the value of the cost. This term can in turn be expressed in state-feedback form, and acts as a degree of freedom that can be employed to stabilise the closed-loop even in cases in which no stabilising solutions exists of the Riccati equation.

\section{References}

[1] H. Abou-Kandil, G. Freiling, V. Ionescu and G. Jank. Matrix Riccati Equations in Control and Systems Theory. Birkhäuser, Basel, 2003.

[2] P. Colaneri and A. Ferrante. Algebraic Riccati Equation and $J$-Spectral Factorization for $\mathscr{H}_{\infty}$ Estimation. Syst. Control Lett., 51(5):383-393, 2004.

[3] A. Ferrante. On the structure of the solution of discrete-time algebraic Riccati equation with singular closed-loop matrix. IEEE T. Automat. Contr., 49(11):2049-2054, 2004.

[4] A. Ferrante, and L. Ntogramatzidis, "A unified approach to finitehorizon generalized LQ optimal control problems for discrete-time systems". Linear Algebra Appl., 425(2-3):242-260, 2007.

[5] A. Ferrante, and H.K. Wimmer, "Order reduction of discrete-time algebraic Riccati equations with singular closed-loop matrix". Oper. Matrices, 1(1):61-70, 2007.

[6] V. Ionescu and C. Oară. Generalized discrete-time Riccati theory. SIAM J. Control Optim., 34(2):601-619, 1996.

[7] V. Ionescu, C. Oară, and M. Weiss. Generalized Riccati theory and robust control, a Popov function approach. Wiley, 1999.

[8] P. Lancaster and L. Rodman. Algebraic Riccati equations. Clarendon Press, Oxford, 1995.

[9] L. Ntogramatzidis, "Self-bounded output-nulling subspaces for non strictly proper systems and their application to the disturbance decoupling problem". IEEE T. Automat. Contr., 53(1):423-428, 2008.

[10] D. Rappaport and L.M. Silverman. Structure and stability of discretetime optimal systems. IEEE T. Automat. Contr., 16:227-233, 1971.

[11] A. Saberi, P. Sannuti, and B.M. Chen. $\mathrm{H}_{2}$ Optimal Control. System and Control Engineering. Prentice Hall International, London, 1995.

[12] A.A. Stoorvogel and A. Saberi. The discrete-time algebraic Riccati equation and linear matrix inequality. Linear Algebra Appl., 274:317$365,1998$.

[13] H.L. Trentelman, A.A. Stoorvogel, and M. Hautus. Control theory for linear systems. Comm. and Control Engineering. Springer, 2001.

[14] H. K. Wimmer. Unmixed Solutions Of The Discrete-Time Algebraic Riccati Equation SIAM J. Control and Optimization, 30(4):867-878, 1992. 